\title{
Das professionelle Rollenverständnis von Lehramtsstudierenden im Verlauf des Studiums: Ein personenzentrierter Ansatz
}

\author{
Jennifer Paetsch, Daniel Mann, Donata Mehler, Barbara Drechsel \\ Otto-Friedrich-Universität Bamberg
}

Zusammenfassung: In der vorliegenden Studie werden sechs verschiedene Facetten des professionellen Rollenverständnisses zum Beruf der Lehrkraft im Verlauf des Studiums anhand eines Mehrkohortendesigns an 1319 Lehramtsstudierenden untersucht. Die Befragten schätzten alle Rollen (Wissensvermittler/-vermittlerin, Mediator/Mediatorin für selbstständige Lernprozesse, Erzieher/ Erzieherin, Helfer/Helferin, Berater/Beraterin, Beurteiler/Beurteilerin) durchschnittlich als wichtig bis sehr wichtig ein. Ein Vergleich von Studierenden zu Beginn und am Ende des Studiums unter Berücksichtigung des Geschlechts und der Schulform zeigte für Mediator/Mediatorin und Beurteiler/ Beurteilerin signifikant höhere Zustimmungen am Ende des Studiums. Mittels latenter Profilanalyse konnten drei Subgruppen von Studierenden identifiziert werden, die sich im Niveau der Zustimmung zu allen Rollenfacetten voneinander unterscheiden. Im anschließenden Gruppenvergleich konnten für Studiendauer und Anzahl der Pflichtpraktika keine signifikanten Unterschiede zwischen den Profilen nachgewiesen werden. Effekte zeigten sich für Geschlecht, studierte Schulform sowie für zusätzliche praktische Erfahrungen und deren Reflexion.

Schlüsselbegriffe: Lehrkräfterolle, Lehramtsstudierende, Überzeugungen, Latente Profilanalyse

\section{Changes in Student Teachers'Beliefs about the Role of Teachers during Initial Teacher Education: A Person-Centered Approach}

Summary: This study focused changes in student teachers' beliefs about the role of teachers during initial teacher education. We investigated six role facets in a multi-cohort-sample of 1319 student teachers while using Latent-Profile-Analysis (LPA). Results reveal that student teachers consider all six role facets (conveyor of knowledge, mediator, pedagogue, helper, counselor, evaluator) as important or very important. Furthermore, results show differences between beginning student teachers and students at the end of their study for the roles mediator and evaluator, under control of school type and gender. Three profiles of different agreement-levels were identified using LPA. Even though we found no group differences for study duration, and number of internships, there were effects for type of school, additional practical experiences and reflection of it.

Keywords: Role of teachers, student teachers, beliefs, Latent-Profile-Analysis

Der Lehrkräfteberuf ist durch eine Vielfalt an Aufgaben gekennzeichnet, die sehr umfangreich in verschiedenen Kontexten beschrieben wurden (Bönsch, 1994; Rothland \& Terhart, 2007; Weiß, Kollmannsberger \& Kiel, 2013).
Eine aktuelle und allgemein akzeptierte Konkretisierung der Aufgabenfelder von Lehrkräften stellen die von der Kultusministerkonferenz verabschiedeten Standards dar, die mit unterschiedlichen Lehrkräfterollen (z. B. Wis-

\section{Förderhinweis:}

Das diesem Artikel zugrunde liegende Vorhaben wird im Rahmen der gemeinsamen „Qualitätsoffensive Lehrerbildung“ von Bund und Ländern mit Mitteln des Bundesministeriums für Bildung und Forschung unter dem Förderkennzeichen 01JA1915 gefördert. Die Verantwortung für den Inhalt dieser Veröffentlichung liegt bei den Autor*innen. 
sensvermittler und -vermittlerin, Erzieher und Erzieherin) verknüpft sind (KMK, 2000; 2019).

Ein Ziel der ersten Phase der Lehrerbildung ist es, Studierende darin zu unterstützen, ihre berufsrelevanten Vorstellungen und Wertorientierungen an diese Standards des Berufsfeldes anzupassen und eine professionelle Identität als Lehrkraft zu entwickeln (vgl. Messner \& Reusser, 2000). Der Entwicklung von berufsbezogenen Überzeugungen wird dabei eine große Bedeutsamkeit für die Nutzung der Lernangebote im Studium, aber auch für das professionelle Handeln als Lehrkraft zugeschrieben (z. B. Calderhead, 1996; Fives \& Buehl, 2012; Reusser $\&$ Pauli, 2014).

Während zu epistemologischen und lehrlerntheoretischen Überzeugungen von (angehenden) Lehrkräften bereits eine Vielzahl an empirischen Forschungsergebnissen vorliegt (z. B. Fives \& Buehl, 2012; Merk, Schneider, Bohl, Kelava \& Syring, 2017; Staub \& Stern, 2002), ist über ihre subjektiven Überzeugungen zur Lehrkräfterolle bislang nur sehr wenig bekannt. Erkenntnisse über das berufliche Rollenverständnis angehender Lehrkräfte und dessen Entwicklung können dazu beitragen, Studierende darin zu unterstützen, eine realistische Vorstellung von ihrem zukünftigen Beruf zu entwickeln (Löfström \& Poom-Valickis, 2013).

Der vorliegende Beitrag knüpft hier an und untersucht verschiedene Facetten des professionellen Rollenverständnisses zum Beruf der Lehrkraft von Lehramtsstudierenden im Verlauf des Studiums anhand eines Mehrkohortendesigns. Ein personenzentrierter Ansatz wurde gewählt, um Subgruppen von Studierenden zu identifizieren, die sich im Verhältnis verschiedener Facetten des professionellen Rollenverständnisses unterscheiden. Da vermutet wird, dass das Rollenverständnis mit individuellen Voraussetzungen und Erfahrungen verbunden ist, wird zudem der Frage nachgegangen, ob sich Unterschiede zwischen den identifizierten Typen auch in Hinblick auf ihre Hintergrundmerkmale oder in ihren Praxiserfahrungen nachweisen lassen.

\section{Überzeugungen als Komponente professioneller Handlungskompetenz von Lehrkräften}

Überzeugungen stellen neben Wissen, der Fähigkeit zur Selbstregulation und motivationalen Orientierungen einen essenziellen Aspekt professioneller Kompetenz von (angehenden) Lehrkräften dar (Baumert \& Kunter, 2006). Lehrkräfteüberzeugungen wird eine wichtige Rolle für das Lehrkräftehandeln zugeschrieben, da sie die Wahrnehmung von Situationen und das didaktische und kommunikative Handeln im Unterricht beeinflussen (vgl. Calderhead, 1996; Pajares, 1992; Philipp, 2007; Woolfolk Hoy, Davis \& Pape, 2009). Studien zeigen zudem Zusammenhänge zwischen Lehrkräfteüberzeugungen und dem Lernerfolg von Schülerinnen und Schülern (Dubberke, Kunter, McElvany, Brunner \& Baumert, 2008; Staub \& Stern, 2002).

Überzeugungen (engl. beliefs) werden als „nicht-wissenschaftliche Vorstellungen darüber, wie etwas beschaffen ist oder wie etwas funktioniert" (Oser \& Blömeke, 2012, S. 415), definiert, die „Bewertungen von und Reaktionen auf Sachverhalte, Situationen und Personen beeinflussen" (Fischer, Hammer \& Ehmke, 2018, S. 155). Geprägt sind Überzeugungen durch persönliche Erfahrungen, die zu Beginn der Lehrkräfteausbildung insbesondere die eigene schulische Biografie betreffen (vgl. Hagemann \& Rose, 1998; Rothland \& Terhart, 2007). Diese intuitiven Überzeugungen stellen jedoch keineswegs professionelle berufsbezogene Überzeugungen dar, die reflektiert, begründet und im Einklang mit wissenschaftlichen Erkenntnissen sind (Pajares, 1992; Reusser \& Pauli, 2014). Eine der Aufgaben der Lehrkräftebildung ist folglich, Lerngelegenheiten zu bieten, welche die Lehramtsstudierenden zu einer reflektierten Auseinandersetzung mit ihren berufsbezogenen Vorstellungen, ihrem beruflichen Selbstverständnis und ihren zukünftigen Aufgabenbereichen animieren.

Insgesamt ist allerdings nur wenig darüber bekannt, wie sich Überzeugungen bei Lehrkräften herausbilden und inwieweit sie durch Interventionen verändert werden können $(\mathrm{Oser} \&$ 
Blömeke, 2012). In der Literatur wird zwar mehrheitlich darauf hingewiesen, dass Studierende bereits zu Beginn ihrer Ausbildung über relativ stabile, schwer veränderbare berufsbezogene Überzeugungen verfügen, jedoch verweisen vorliegende empirische Studien auf signifikante Zusammenhänge zwischen wahrgenommenen Lernangeboten und Überzeugungen der Studierenden (Biedermann, Brühwiler \& Krattenmacher, 2012; Biedermann, Brühwiler \& Steinmann, 2012; de Vries, Jansen \& van de Grift, 2013; Fischer \& Ehmke, 2019). Dabei liegt ein wechselseitiger Zusammenhang vor: Einerseits sind berufsbezogene Überzeugungen relevant für die Auswahl und das Erleben von Lerngelegenheiten während des Studiums, andererseits scheinen Lerngelegenheiten bedeutsam zur Veränderung von Überzeugungen beizutragen (vgl. Fischer \& Ehmke, 2019). Welche Kontextfaktoren dabei die Herausbildung von professionellen Überzeugungen begünstigen oder behindern, ist bislang nicht abschließend geklärt (vgl. Reusser \& Pauli, 2014). Es wird jedoch angenommen, dass Reflexion und bewusste Auseinandersetzung mit den eigenen Überzeugungen und Erfahrungen wichtige Voraussetzungen sind (vgl. Philip, 2007; Reusser \& Pauli, 2014). Besonders hervorgehoben wird dabei immer wieder die Relevanz von Erfahrungen in der pädagogischen Praxis (vgl. z. B. Korthagen \& Kessels, 1999; Richardson, 1996). So konnte in einer Untersuchung von Keller-Schneider (2018) gezeigt werden, dass sich selbstbezogene Überzeugungen während des Praktikums wandeln. Allerdings weisen die Befunde auf individuell differente Entwicklungsverläufe hin, was bedeutet, dass sich die praktischen Erfahrungen nicht für alle Studierenden auf dieselbe Art und Weise auswirkten (Keller-Schneider, 2018).

Bei der Betrachtung des Forschungsstandes wird deutlich, dass sich professionelle Überzeugungen von Lehrkräften auf verschiedene Gegenstandsbereiche beziehen und unterschiedlich konzeptualisiert werden (z. B. Calderhead, 1996; Pajares, 1992; Woolfolk Hoy et al., 2009). So unterscheiden Reusser und Pauli (2014) zwischen epistemologischen Überzeugungen, per- sonenbezogenen Überzeugungen und kontextbezogenen Überzeugungen. Kunter und Pohlmann (2015) unterscheiden hingegen vier Bezugssysteme (Selbst, Lehr-Lernkontext, Bildungssystem, Gesellschaft) und ordnen diesen Inhalte von Überzeugungen zu (z. B. Vorstellungen zur eigenen Identität, Überzeugungen über Lehren und Lernen).

\section{Das professionelle Rollenverständnis zum Beruf der Lehrkraft}

Das professionelle Rollenverständnis zum Beruf der Lehrkraft ist eine Facette der berufsbezogenen Überzeugungen (angehender) Lehrkräfte, die sich in der Taxonomie von Reusser und Pauli (2014) den personenbezogenen Überzeugungen zuordnen lässt, die „sich auf die Akteure des bildungsbezogenen Handelns im Schulkontext, primär auf die professionsbezogene Selbstwahrnehmung der Lehrkräfte“ (S. 651) beziehen. Sie gehören dabei zum Bezugssystem "Selbst“ im Sinne des selbstbezogenen Rollenverständnisses, lassen sich jedoch auch in den anderen Bezugssystemen im Sinne von allgemeinen Vorstellungen über die Lehrkräfterolle verorten (Kunter \& Pohlmann, 2015).

Ein professionelles Rollenverständnis orientiert sich an den Standards des Berufsfeldes (vgl. Messner \& Reusser, 2000). Dabei ist der Lehrkräfteberuf durch eine Vielfalt an Aufgaben und Rollen gekennzeichnet, die aus theoretischnormativer Perspektive in der Literatur häufig beschrieben wurden (z. B. Bönsch, 1994). Zu einer Erweiterung des Rollenspektrums haben u. a. Erkenntnisse der Lehr-Lernforschung, die Digitalisierung aller Lebensbereiche, eine zunehmende Heterogenität der Schülerschaft und Debatten zur Ökonomisierung der Bildung beigetragen (Reusser, 2000; 2006; Terhart, 2011).

Aktuelle Charakterisierungen der Aufgabenfelder von Lehrkräften, die Ergebnisse empirischer Forschung berücksichtigen (Rothland \& Terhart, 2007; Weiß et al., 2013), liegen mit den von der Kultusministerkonferenz verabschiedeten Standards vor. Hier werden explizit verschiedene Lehrkräfterollen angesprochen: Wis- 
sensvermittler und -vermittlerin, Erzieher und Erzieherin, Beurteiler und Beurteilerin, Berater und Beraterin (KMK, 2000; 2019; vgl. Kunter et al., 2017, S. 86ff). Diese Rollenbeschreibungen gelten zwar für alle Schularten, doch gibt es Hinweise darauf, dass schulartspezifische Anforderungsprofile existieren. Beispielsweise zeigen die Ergebnisse von Weiß et al. (2013), die in Gruppendiskussionen 220 Lehrkräfte mit mindestens 10-jähriger Berufserfahrung in der jeweiligen Schulart befragt haben, dass an Gymnasien die Rolle des Wissensvermittlers bzw. der -vermittlerin als besonders wichtig hervorgehoben wird und im Grund-, Hauptund Realschulbereich besonders Anforderungen gesehen werden, die durch vielfältige Beratungsanlässe entstehen. Schulartunterschiede im Rollenverständnis von (angehenden) Lehrkräften lassen sich auch aufgrund spezifischer Berufswahlmotive vermuten. Beispielsweise ist bekannt, dass angehende Gymnasiallehrkräfte sich im Vergleich zu Studierenden des Grundschullehramts durch ein im Durchschnitt stärkeres fachliches und geringeres pädagogisches Interesse auszeichnen (vgl. z. B. Retelsdorf \& Möller, 2012).

$\mathrm{Zu}$ der Frage, wie angehende Lehrkräfte ihre zukünftige berufliche Rolle einschätzen und wie sich dieses Rollenverständnis während des Studiums verändert, liegen nur wenige empirische Studien vor. Saban, Kocbeker und Saban (2007) haben an 1142 Lehramtsstudierenden in der Türkei untersucht, wie sie die Rolle der Lehrkraft beschreiben. Die genannten Metaphern wurden in 10 Kategorien zusammengefasst. Lehrkräfte wurden von den Studierenden am häufigsten als Wissensanbieterin bzw. -anbieter, als Handwerkerin bzw. Handwerker, als Erzieherin bzw. Erzieher, als Beraterin bzw. Berater beschrieben (Saban et al., 2007). Darüber hinaus zeigte sich, dass Studierende am Beginn des Studiums die Lehrkräfterolle häufiger mit entwicklungsorientierten (z. B. fürsorglich, aufziehend) und Studierende am Ende des Studiums eher mit unterstützend-moderierenden Metaphern (z. B. navigierend, unterstützend) beschreiben (Saban et al., 2007).
Löfström und Poom-Valickis (2013) untersuchten in ihrer Längsschnittstudie, welche Rollenaspekte Studierende Lehrkräften generell zuschreiben. Befragt wurden 80 estländische Studierende (nicht nur Lehramt) zu mehreren Zeitpunkten während des Studiums. Am häufigsten wurde die Rolle der Lehrkraft als Pädagogin bzw. als Pädagoge, als Expertin bzw. Experte für Fachinhalte und als Didaktikerin bzw. Didaktiker beschrieben; es gab keine Unterschiede zwischen den Zeitpunkten (Löfström \& Poom-Valickis, 2013).

Zusammenfassend lässt sich feststellen, dass, obwohl der Übernahme der Lehrkräfterolle im Rahmen der Professionalisierung von angehenden Lehrkräften eine wichtige Bedeutung zugeschrieben wird (KMK, 2019), viele Fragen zu Ausprägung und Entwicklung des professionellen Rollenverständnisses von Lehramtsstudierenden offen sind. Die wenigen vorliegenden Studien lassen erkennen, dass Lehramtsstudierende verschiedene Rollenfacetten als unterschiedlich bedeutsam für ihren Beruf einschätzen und sich dieses Muster im Laufe des Studiums verändern kann.

\section{Fragestellungen}

In der vorliegenden Studie werden nicht nur die Ausprägungen der subjektiven Rollenüberzeugungen von Lehramtsstudierenden untersucht, sondern auch, ob sich diese im Laufe des Studiums verändern. Da sich die Rolle der Lehrkraft zwischen verschiedenen Schularten in einigen Aspekten voneinander unterscheiden kann (z. B. Weiß et al., 2013), und gleichzeitig der Anteil weiblicher Lehramtsstudierender zwischen den Schulformen variiert (z. B. Retelsdorf \& Möller, 2012), werden Geschlecht und der gewählte Studiengang explizit berücksichtigt.

Für die Untersuchung von subjektiven Rollenüberzeugungen scheint zudem eine personenzentrierte Analyse gewinnbringend zu sein, um die Zusammenhänge zwischen verschiedenen Rollenfacetten berücksichtigen und somit typische Merkmalskonfigurationen der Lehr- 
amtsstudierenden identifizieren zu können. Während variablenzentrierte Ansätze Durchschnittswerte der Merkmale und deren Relationen zueinander über die Stichprobe hinweg untersuchen, liefern personenzentrierte Ansätze Informationen über die Merkmalskonfigurationen in der untersuchten Personengruppe, die mit relevanten Variablen in Beziehung gesetzt werden können.

Konkret werden in der vorliegenden Studie drei Fragestellungen untersucht:

1) Unterscheiden sich Facetten des professionellen Rollenverständnisses zwischen Lehramtsstudierenden am Beginn und am Ende des Studiums unter Berücksichtigung des Studiengangs (Schulform) und des Geschlechts?

In Hinblick auf die Entwicklung eines professionellen Rollenverständnisses werden Unterschiede in die Richtung erwartet, dass alltagstheoretisch antizipierte Vorstellungen zur Lehrkräfterolle, wie z. B. Wissensvermittlung und Beurteilung, an Wichtigkeit verlieren, wohingegen Aufgabenfelder von Lehrkräften, die vermutlich erst im Studium explizit bewusst werden, wie z. B. das Beraten und das Koordinieren selbstständiger Lernprozesse, eine Aufwertung erfahren.

\section{2) Lassen sich innerhalb einer Stichprobe von Lehramtsstudierenden verschiedene Subgruppen mit unterschiedlichen Rollen- profilen identifizieren?}

Es wird angenommen, dass sich aufgrund von unterschiedlichen Lerngelegenheiten und persönlichen Voraussetzungen Gruppen von Lehrkräften identifizieren lassen, die ähnliche Rollenprofile aufweisen, das heißt, bei denen bestimmte Rollenfacetten hoch und andere niedrig ausgeprägt sind. Da bislang nur wenig über subjektive Rollenüberzeugungen von Lehramtsstudierenden bekannt ist, werden hier keine Hypothesen zu vorliegenden Subgruppen formuliert.
In einem letzten Schritt soll schließlich untersucht werden, ob sich die gefundenen Subgruppen hinsichtlich relevanter Variablen unterscheiden. Ziel ist es, Einblicke in die Entstehung von subjektiven Rollenüberzeugungen zu erhalten:

\section{3) Unterscheiden sich die identifizierten} Subgruppen in ihrer Zusammensetzung systematisch in Bezug auf relevante Hintergrundmerkmale (Studiendauer, Studiengang, Geschlecht) und hinsichtlich ihrer bisherigen praktischen Erfahrungen im pädagogischen Kontext?

Insbesondere Unterschiede in Hinblick auf Studiendauer und praktische Erfahrungen könnten Hinweise auf Veränderungen von Rollenüberzeugungsmustern während des Studiums liefern. Aus dem Forschungsstand zur Veränderbarkeit von Überzeugungen lässt sich ableiten, dass insbesondere intensiv reflektierte praktische Erfahrungen bei der Veränderung von Überzeugungen relevant sein können (z. B Philipp, 2007).

\section{Methode}

\section{Untersuchungsdesign und Stichprobe}

Die Daten der vorliegenden Studie wurden im Rahmen des Projekts "Wegweisende Lehrerbildung“ (WegE, Qualitätsoffensive Lehrerbildung) in einem querschnittlichen Mehrkohortendesign an der OttoFriedrich-Universität Bamberg erhoben. Der Befragungszeitraum begann im Wintersemester 2016/17 und endete im Wintersemester 2018/19. Lehramtsstudierende unterschiedlicher Studiendauer wurden in diesem Zeitraum zu insgesamt fünf Zeitpunkten (in jedem Semester) rekrutiert, um an einer Befragung teilzunehmen. Diese fanden in Präsenzveranstaltungen statt. Zusätzlich wurden Studierende mit fortgeschrittener Studiendauer direkt nach dem Absolvieren einer Staatsexamensprüfung gebeten, an der Befragung teilzunehmen. Weiterhin gab es eine Online-Befragung, zu deren Teilnahme Lehramtsstudierende mit fortgeschrittener Studiendauer eingeladen wurden. 
Tab. 1: Stichprobenzusammensetzung und Kohortenzuordnung nach Studienfortschritt

\begin{tabular}{|c|c|c|c|c|c|c|}
\hline & \multirow{2}{*}{\multicolumn{3}{|c|}{ Kohorte }} & & \\
\hline & & & & & \multirow{3}{*}{ gesamt } & \multirow{3}{*}{$\%_{\text {weiblich }}$} \\
\hline & & 1 & 2 & 3 & & \\
\hline \multicolumn{2}{|l|}{ Semester } & $1 .-2$ & $3 .-5$ & 6.- max. & & \\
\hline Anzahl & $\begin{array}{l}n \\
\%_{\text {Stichprobe }} \\
\%_{\text {weiblich }}\end{array}$ & $\begin{array}{c}507 \\
38,9 \% \\
84,0 \%\end{array}$ & $\begin{array}{c}380 \\
29,2 \% \\
82,9 \%\end{array}$ & $\begin{array}{c}415 \\
31,9 \% \\
81,7 \%\end{array}$ & $\begin{array}{c}1302 \\
100,0 \% \\
82,9 \%\end{array}$ & \\
\hline Schulart (Anzahl) & $\begin{array}{l}\text { Grundschule } \\
\text { Mittel-/Realschule } \\
\text { Gymnasiums }\end{array}$ & $\begin{array}{l}261 \\
137 \\
109\end{array}$ & $\begin{array}{r}244 \\
71 \\
65\end{array}$ & $\begin{array}{r}203 \\
63 \\
149\end{array}$ & $\begin{array}{l}708 \\
271 \\
323\end{array}$ & $\begin{array}{l}92,1 \% \\
72,7 \% \\
71,5 \%\end{array}$ \\
\hline
\end{tabular}

Anmerkungen: Bei $n=17$ Personen ist die Studiendauer aufgrund fehlender Angaben nicht bekannt.

Die Stichprobe besteht insgesamt aus 1319 Lehramtsstudierenden ${ }^{1}$. Die Studierenden verteilen sich wie folgt auf die Schularten - Grundschule: 54,2\%, Mittelschule/Realschule: 20,9\%, Gymnasium: 24,9\%. Die Teilnehmenden befanden sich durchschnittlich im vierten Semester $(M=4.20, S D=3.57)$ und waren im Mittel 22 Jahre alt $(M=21.53, S D=3.18)$. Die Mehrheit der Teilnehmenden (82,9\%) ist weiblich. Um Studierende zu identifizieren, die am Beginn bzw. am Ende ihres Studiums sind, wurde die Stichprobe anhand der Studiendauer in drei Gruppen eingeteilt (s. Tabelle 1).

Die Angaben zu den praktischen Erfahrungen basieren designbedingt auf Teilstichproben, da nicht alle Befragungen Angaben zu den bisherigen Praxiserfahrungen enthielten ${ }^{2}: N_{\text {pfichtpraktika }}=302(84,8 \%$ weiblich, 55,6\% Grundschule, 16,6\% Mittelschule/ Realschule, 27,8\% Gymnasium), $N_{\text {zușätliche Praktika }}=428$ (83,2\% weiblich, 52,8\% Grundschule, $15,7 \%$ Mittelschule/Realschule, 31,5\% Gymnasium).

\section{Instrumente}

Zur Erfassung des auf den Lehrkräfteberuf bezogenen professionellen Rollenverständnisses wurde ein aus vier Skalen bestehendes Instrument der BilWiss-Studie (Kunter et al., 2017) um zwei weitere Skalen ergänzt. Aus der BilWiss-Studie stammen die vier Skalen Wissensvermittler/-vermittlerin (WV), Mediator/Mediatorin für selbstständige Lernprozesse (ML), Erzieher/Erzieherin (ER) und Helfer/Helferin (HE) (ebd., S. 86ff). Auf Grundlage der KMK-Standards für die Lehrerbildung $(2019)^{3}$ wurden die Skalen zu den beiden Rollen Berater/Beraterin (BER) und Beurteiler/Beurteilerin (BEU) neu entwickelt. Erfasst wurde die Zustimmung zu den jeweiligen rollenbezogenen Tätigkeiten einer
Lehrkraft (Wie wichtig sind für Sie persönlich folgende Aufgaben einer Lehrkraft?) auf einer sechsstufigen Antwortskala von sehr unwichtig (1) bis sehr wichtig (6) (Beispielitems siehe Tabelle 2). Insgesamt besteht das Instrument aus 36 Items. Die Reliabilitäten der eingesetzten Skalen liegen zwischen $\alpha=.71$ und $\alpha=.87$ und sind entsprechend als befriedigend bis gut zu bewerten (s. Tabelle 2). Die bivariaten Korrelationen der Rollenfacetten liegen zwischen $r=.24$ und $r=.53(p<.01)$.

Die Studierenden wurden nach bisher absolvierten Pflichtpraktika ${ }^{4}$ im Studium sowie nach zusätzlichen Praxiserfahrungen gefragt (Welche Praxiserfahrungen [wie z. B. Nachhilfe oder Mittagsbetreunng] haben Sie zusätzlich zu Ihren Pflichtpraktika gemacht?). Außerdem wurde von den Studierenden, auf einer vierstufigen Skala von trifft nicht $z u$ (1) bis trifft $z u$ (4), eine Einschätzung erbeten, wie hilfreich sie diese zusätzlichen Praxiserfahrungen fanden (Diese Erfahrung hilft mir zur Reflexion und Anwendung meines Theoriewissens.).

1 Bei Mehrfachteilnahme von Personen innerhalb des Untersuchungszeitraumes wurde nur die letzte Messung berücksichtigt, da Studierende in höheren Semestern weniger häufig in der Stichprobe vertreten waren. Im Wintersemester 2018/19 waren 2057 Studierende im Lehramt an der Otto-Friedrich-Universität Bamberg eingeschrieben.

2 In Befragungen von Studierenden in den ersten Semestern wurde auf die Angabe von praktischen Erfahrungen verzichtet. Aus diesem Grund bestehen die Teilstichproben aus Studierenden in höheren Semestern, die bereits Pflichtpraktika absolviert haben.

3 Kompetenzbereich: Beurteilen („Lehrkräfte beraten sach- und adressatenorientiert und üben ihre Beurteilungsaufgabe gerecht und verantwortungsbewusst aus.", KMK, 2019, S. 11)

4 Orientierungspraktikum, pädagogisch-didaktisches Schulpraktikum, studienbegleitendes fachdidaktisches Praktikum im Fach, zusätzliches studienbegleitendes fachdidaktisches Praktikum im Fach 


\section{Statistische Verfahren}

Die Prüfung der ersten Fragestellung erfolgte mit 3-faktoriellen Varianzanalysen. Die Werte waren nicht normalverteilt für die Gruppen, wie ShapiroWilk-Tests ergaben. Zudem waren die Gruppen sehr unterschiedlich groß (durch die vergleichsweise geringe Anzahl an männlichen Studierenden). Aus diesem Grund wurden die Ergebnisse der ANOVA anhand der durch ein Bootstrapping generierten BCa-Intervalle (bias corrected and accelerated) validiert (vgl. Hesterberg, 2015).

Ein Ziel der vorliegenden Studie stellt die Bildung von Subgruppen dar, die auf Basis von latenten Profilanalysen (LPA) erfolgte. Die Datengrundlage bildeten die erhobenen Ausprägungen der erfassten sechs Lehrkräfterollen. LPA stellen eine personenzentrierte Strategie der Datenanalyse dar (vgl. Rost, 2004). Personen werden aufgrund ihrer Antwortmuster zu bestimmten latenten Profilen zugeordnet.
Dabei ist es das Ziel, die kleinstmögliche Anzahl latenter Profile zu identifizieren, denen möglichst alle Fälle mit hoher Wahrscheinlichkeit zugeordnet werden können. Zur Bewertung des Modellfits werden folgende Indikatoren herangezogen: BIC, Likelihood-Differenztests (VLMR; Lo, Mendell \& Rubin, 2001; sowie BLRT) und Entropie. Die statistischen Kennwerte stellen allerdings nur Anhaltspunkte zur Auswahl der endgültigen Anzahl latenter Profile dar. Die Entscheidung für eine Lösung sollte auch auf der Grundlage inhaltlich-theoretischer Gesichtspunkte erfolgen (vgl. Alexandrowicz, 2008).

Die LPA wurde mit der Software Mplus 8.3 (Muthén \& Muthén, 1998-2019) durchgeführt. Fehlende Werte wurden anhand der „Full Information Maximum Likelihood (FIML)"-Methode bei der Schätzung berücksichtigt. Die Prüfung auf Unterschiede zwischen Gruppen erfolgte für metrische Daten mit Varianzanalysen und für kategoriale Daten mittels Chi-Quadrat-Test in SPSS.

Tab. 2: Deskriptive Statistik: Wichtigkeit von Rollen einer Lehrkraft \& Angaben zu praktischen Erfahrungen

\begin{tabular}{|c|c|c|c|c|c|c|c|c|c|}
\hline Skala & Abk. & $n_{\text {Items }}$ & $a$ & $n$ & $n_{\text {fehlend }}$ & $M$ & $S D$ & Min & Max \\
\hline $\begin{array}{l}\text { Wissensvermittlerl-vermittlerin } \\
\text { (z.B. Vielseitiges Wissen an die } \\
\text { Schüler(innen) weitergeben.) }\end{array}$ & WV & 9 & .78 & 1287 & 32 & 5.05 & 0.47 & 1.44 & 6.00 \\
\hline $\begin{array}{l}\text { Mediator/Mediatorin für Lernprozesse } \\
\text { (z. B. Die Schüler(innen) zum Lernen } \\
\text { zu motivieren.) }\end{array}$ & $M L$ & 6 & .77 & 1307 & 12 & 5.42 & 0.50 & 2.17 & 6.00 \\
\hline $\begin{array}{l}\text { Erzieher/Erzieherin } \\
\text { (z.B. Die Schüler(innen) zu selbst- } \\
\text { ständigen Persönlichkeiten zu erziehen.) }\end{array}$ & ER & 7 & .84 & 1298 & 21 & 5.27 & 0.60 & 1.71 & 6.00 \\
\hline $\begin{array}{l}\text { Helfer/Helferin } \\
\text { (z.B. Als Ansprechpartner(in) für } \\
\text { persönliche Probleme zur Verfügung } \\
\text { zu stehen.) }\end{array}$ & HE & 5 & .87 & 1309 & 10 & 5.15 & 0.71 & 1.40 & 6.00 \\
\hline $\begin{array}{l}\text { Berater/Beraterin } \\
\text { (z.B.Schüler(innen) hinsichtlich ihrer } \\
\text { Lernprozesse beraten.) }\end{array}$ & BER & 6 & .80 & 1298 & 21 & 4.98 & 0.62 & 1.00 & 6.00 \\
\hline $\begin{array}{l}\text { Beurteiler/Beurteilerin } \\
\text { (z.B.Schulische Leistungen auf der } \\
\text { Grundlage transparenterBeurteilungs- } \\
\text { maßstäbe beurteilen.) }\end{array}$ & BEU & 3 & .71 & 1309 & 10 & 4.71 & 0.74 & 1.33 & 6.00 \\
\hline $\begin{array}{l}\text { Anzahl Pflichtpraktika } \\
\text { Anzahl zusätzlicher Praxiserfahrungen } \\
\text { Reflexion zusätzlicher Praxiserfahrung } \\
\text { Index: zusätzliche Praxiserfahrungen } \times \text { Ref }\end{array}$ & $\begin{array}{l}\text { en } \\
\text { flexion }\end{array}$ & $\begin{array}{l}- \\
- \\
3 \\
-\end{array}$ & $\begin{array}{l}- \\
- \\
78 \\
-\end{array}$ & $\begin{array}{l}302 \\
428 \\
305 \\
418\end{array}$ & $\begin{array}{l}- \\
10 \\
- \\
10\end{array}$ & $\begin{array}{l}4.12 \\
1.19 \\
3.03 \\
3.60\end{array}$ & $\begin{array}{l}0.95 \\
0.94 \\
0.95 \\
3.15\end{array}$ & $\begin{array}{l}0.00 \\
0.00 \\
1.00 \\
0.00\end{array}$ & $\begin{array}{r}5.00 \\
3.00 \\
4.00 \\
12.00\end{array}$ \\
\hline
\end{tabular}

Anmerkungen: $n_{\text {Items }}=$ Anzahl der Items; $\alpha=$ Cronbachs Alpha; $n=$ Fallzahl; $n_{\text {fehlend }}=$ Missings; $M=$ Mittelwert $S D=$ Standardabweichung; Min = Minimum; Max = Maximum. 


\section{Ergebnisse}

\section{Deskriptive Ergebnisse}

Die Befragten schätzten alle Rollen durchschnittlich als wichtig bis sehr wichtig ein (s. Tabelle 2). Dabei weist die Rolle BEU den niedrigsten Mittelwert $(M=4.71, S D=0.74)$ und die Rolle ML den höchsten $(M=5.42, S D=0.50)$ auf. Die geringste Varianz zeigte sich bei der Rolle WV $(S D=0.47)$, die größte Varianz hinsichtlich der Rolle BEU ( $S D=0.74)$. Die Studierenden der Teilstichprobe (s.o.) absolvierten durchschnittlich 4.12 Pflichtpraktika $(S D=0.95)$ und im Mittel 1.19 zusätzliche Praxiserfahrungen $(S D=0.94)$. Die Frage nach Reflexion und Anwendung der zusätzlichen Praxiserfahrungen wurde auf der vierstufigen Skala im Durchschnitt mit $M=3.03$ beantwortet $(S D=0.95)$. Es wurde ein multiplikativer Index aus den angegebenen zusätzlichen Praxiserfahrungen und der darauf bezogenen Antworten zur Reflexion gebildet (s. Tabelle 2). Der Index setzte Merkmalsausprägungen auf beiden Indikatorvariablen voraus: Auch viele zusätzliche Praxiserfahrungen können eine fehlende Reflexion dieser nicht kompensieren.

\section{Das professionelle Rollenverständnis zu Beginn und am Ende des Studiums}

Die erste Fragestellung wurde anhand von 3-faktoriellen Varianzanalysen mit den Faktoren Kohorte (1/3), Geschlecht (m/w) und Studiengang (Grundschule, Realschule/Mittelschule, Gymnasium) überprüft (s. Tabelle 3). Signifikante Ergebnisse wurden anhand der durch ein Bootstrapping generierten $\mathrm{BCa}$-Intervalle validiert.

Für die Rolle WV verfehlte der Haupteffekt Kohorte knapp das Signifikanzniveau und auch für die Schulart zeigte sich kein signifikanter Effekt. Gefundene Geschlechtsunterschiede ließen sich durch die BCa-Intervalle nicht bestätigen. Es lag kein signifikanter Interaktionseffekt vor.

Erwartungskonform zeigte sich, dass die Rolle ML von Kohorte 3 für signifikant wichtiger gehalten wurde als von Kohorte $1(F(1,901)=$ $\left.10.97, p<.01, \eta^{2}=.01\right)$. Auch für die verschiedenen Schularten war ein signifikanter Haupt- effekt nachweisbar $(F(2,901)=5.86, p<.01$, $\eta^{2}=.01$ ). Kontraste zeigten, dass Studierende des Grundschullehramts diese Rolle im Vergleich zu Studierenden des Mittel- bzw. Realschullehramts und des Gymnasiallehramts signifikant wichtiger einschätzten, mit einer durchschnittlichen Differenz von $0.36(\mathrm{SE}=0.10, p<.01$, Bonferronikorrigiert). Darüber hinaus zeigten weibliche Studierende signifikant höhere Einschätzungen als ihre männlichen Kommilitonen $(F(1,901)=$ 7.47, $\left.p<.01, \eta^{2}=.01\right)$. Es lag kein signifikanter Interaktionseffekt vor.

Für die Rolle ER konnten signifikante Haupteffekte für Kohorte, Schulart und Geschlecht nicht durch BCa-Intervalle bestätigt werden. Es gab keine signifikante Interaktion.

Für die Rolle HE war kein signifikanter Haupteffekt für die Kohorte nachweisbar, jedoch für die Schulart $(F(2,903)=15.18, p<.001$, $\left.\eta^{2}=0.03\right)$. Kontraste zeigten, dass Studierende des Grundschullehramts diese Rolle im Vergleich zu Studierenden des Mittel- bzw. Realschullehramts und des Gymnasiallehramts signifikant wichtiger einschätzten, mit einer durchschnittlichen Differenz von 0.63 ( $\mathrm{SE}=0.15, p<.01$, Bonferroni-korrigiert). Darüber hinaus zeigten weibliche Studierende signifikant höhere Einschätzungen als ihre männlichen Kommilitonen $\left(F(1,903)=31.26, p<.01, \eta^{2}=.03\right)$. Es gab eine statistisch signifikante Interaktion zwischen Geschlecht und Schulart $(F(2,903)=5.17, p<.01$, $\left.\eta^{2}=.01\right)$ : Geschlechtsunterschiede waren nur für Gymnasialstudierende nachzuweisen und der Unterschied zwischen Grundschule und Gymnasium war nur bei männlichen Studierenden signifikant.

Für die Rolle BER war kein signifikanter Haupteffekt für die Kohorte nachweisbar, jedoch für die Schulart $(F(2,897)=7.96, p<.001$, $\left.\eta^{2}=.02\right)$. Studierende des Grundschullehramts schätzten diese Rolle im Vergleich zu Studierenden des Mittel- bzw. Realschullehramts und des Gymnasiallehramts signifikant wichtiger ein, mit einer durchschnittlichen Differenz von 0.45 ( $\mathrm{SE}=0.13, p<.001$, Bonferroni-korrigiert). Darüber hinaus zeigten weibliche Studierende signifikant höhere Einschätzungen als ihre männ- 
=

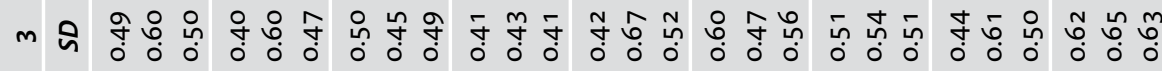

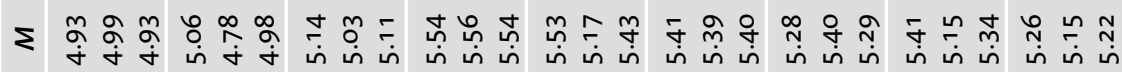

$\stackrel{ \pm}{0}$
$\stackrel{ \pm}{0}$
$\stackrel{ \pm}{0}$

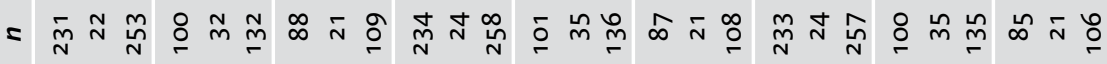

-

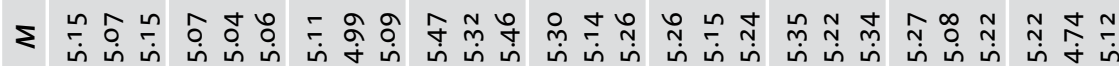

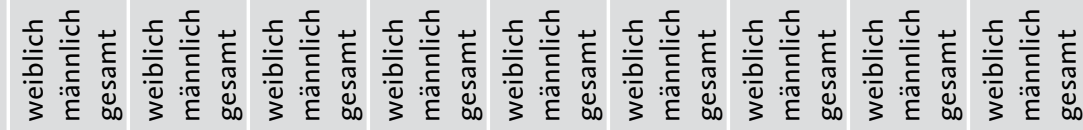

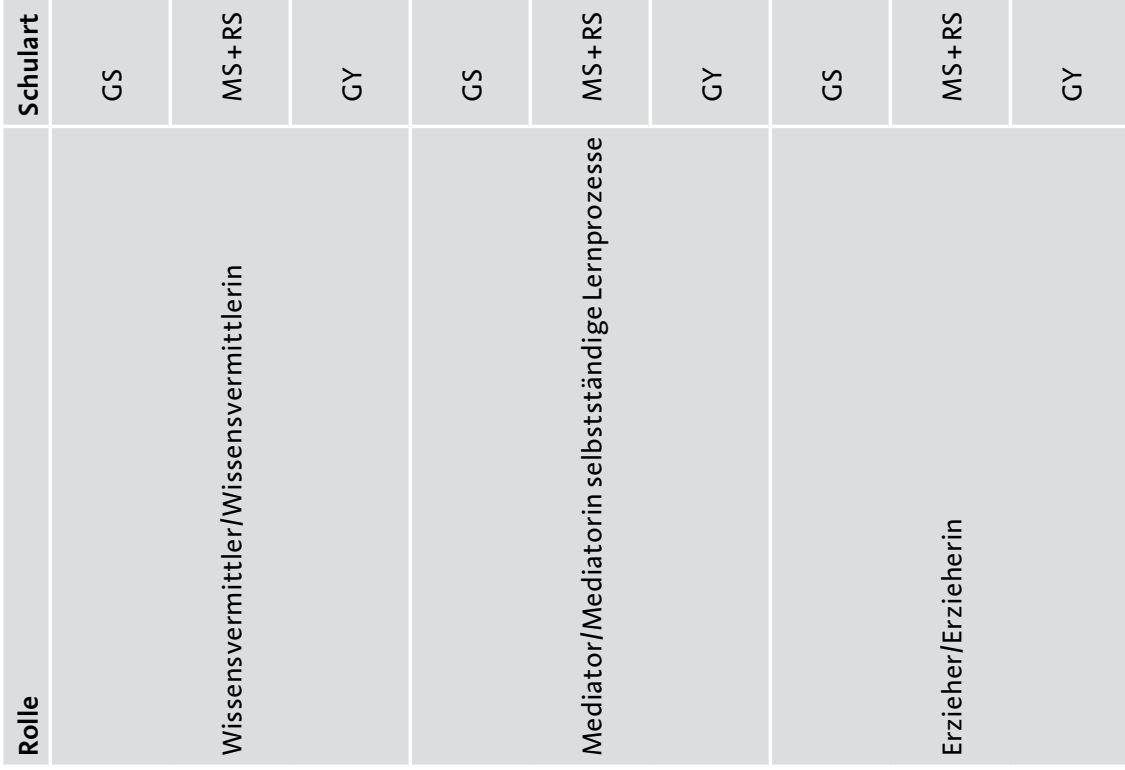




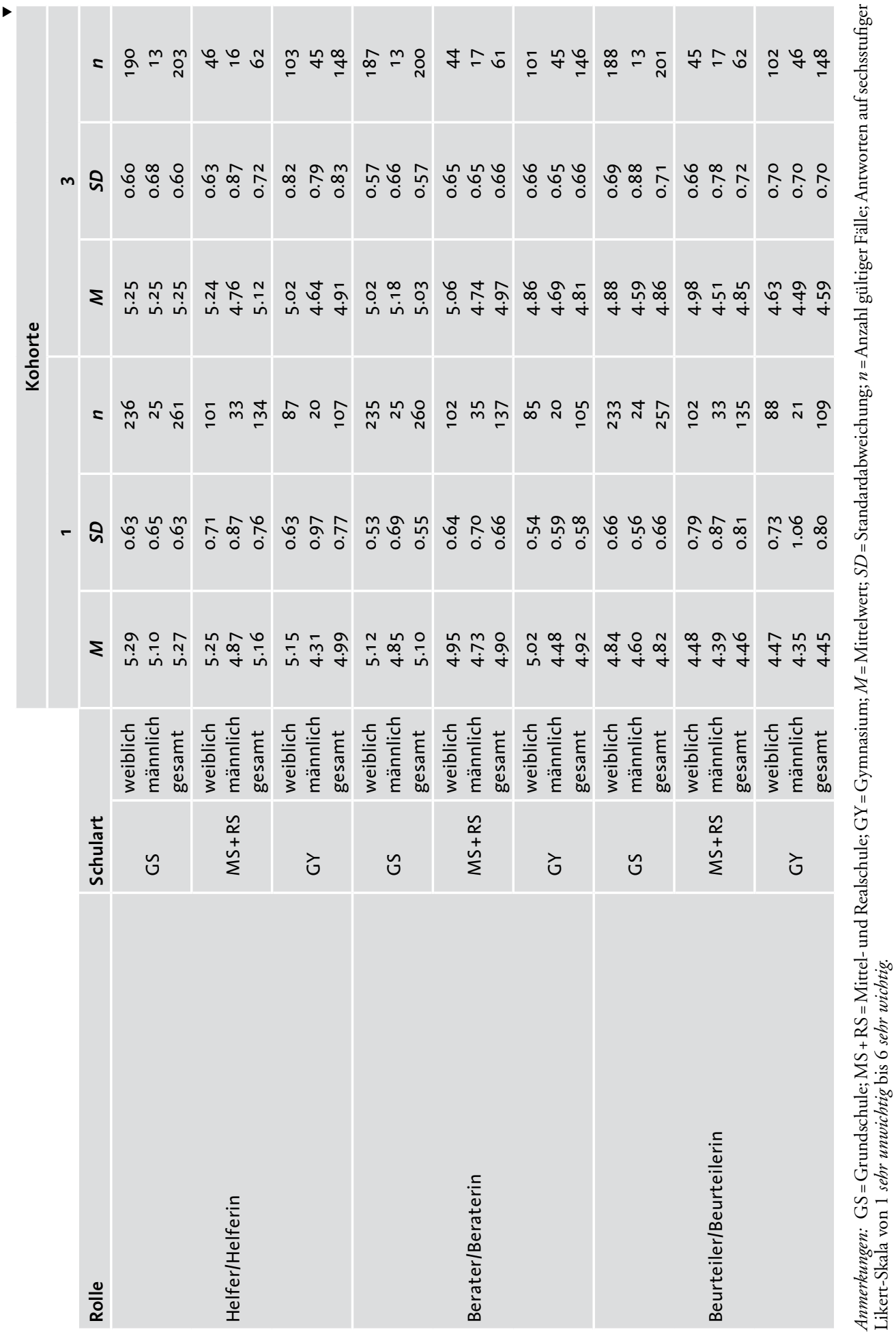




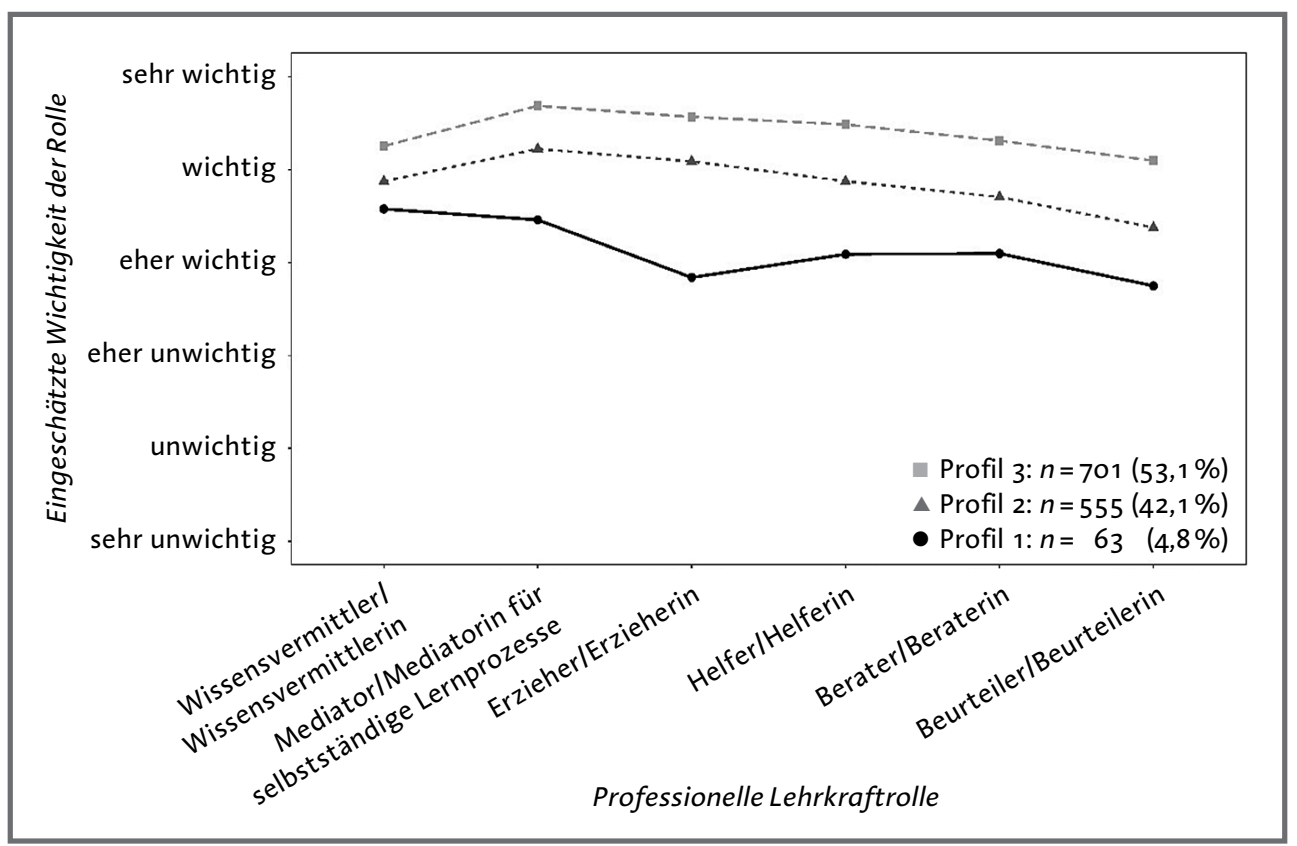

Abb. 1: Darstellung der drei Profile über die sechs Skalen des Rollenverständnisses bei Lehramtsstudierenden

lichen Kommilitonen $(F(1,897)=15.50, p<.01$, $\left.\eta^{2}=.02\right)$. Es gab eine statistisch signifikante Interaktion zwischen Geschlecht und Kohorte $\left(F(1,897)=3.88, p<.05, \eta^{2}<.01\right)$ : Von männlichen Studierenden wurde die Rolle BER am Ende des Studiums wichtiger eingeschätzt als zu Beginn, bei den weiblichen Studierenden war es umgekehrt.

Die Rolle BEU wurde von Kohorte 3 als wichtiger eingeschätzt als von Kohorte $1(F(1,900)=$ $\left.5.08, p<.05, \eta^{2}=.01\right)$. Auch für die verschiedenen Schularten war ein signifikanter Haupteffekt nachweisbar $(F(2,900)=4.18, p<.05$, $\left.\eta^{2}=.01\right)$ : Studierende des Grundschullehramts schätzten diese Rolle im Vergleich zu Studierenden des Mittel- bzw. Realschullehramts und des Gymnasiallehramts mit einer durchschnittlichen Differenz von 0.38 ( $\mathrm{SE}=0.15, p<.05$, Bonferroni-korrigiert) signifikant wichtiger ein. Darüber hinaus zeigten weibliche Studierende signifikant höhere Einschätzungen als ihre männlichen Kommilitonen $(F(1,900)=10.53$, $\left.p<.01, \eta^{2}=.01\right)$. Es gab keine signifikante Interaktion.

\section{Profile des Rollenverständnisses}

Zur Identifikation von Subgruppen (Fragestellung 2) wurden in einer LPA die Einschätzungen der Studierenden zu den sechs Rollen einer Lehrkraft genutzt (s. Tabelle 2). Die statistischen Kennwerte werden in Tabelle 4 angegeben. AIC, BIC und aBIC wurden mit zunehmender Anzahl latenter Profile durchweg kleiner, sodass sie auf keine spezifische Lösung hinwiesen. Der BLRT nahm für alle Zahlen latenter Profile signifikante Werte an. Der VLMRTest verwies darauf, dass eine Zwei-Profillösung besser zu den Daten passt als eine Ein-Profillösung. Ebenfalls zeigte der VLMRT, dass die Lösung mit drei Profilen eine tendenziell bessere Modellgüte besaß als die Lösung mit zwei Profilen. Werden zu den statistischen Kriterien des Modellvergleichs auch inhaltlich-theoriebasierte Kriterien herangezogen, so besaß die Lösung mit drei Profilen neben vertretbarer statistischer Modellgüte die beste Interpretierbarkeit im Vergleich zu den anderen Modelllösungen (vgl. Abbildung 1). Daher wurde die Drei- 
Tab. 4: Fitindizes der Modellgüte für Lösungen mit 2 bis 5 latenten Profilen

\begin{tabular}{|l|l|l|l|l|l|l|c|}
\hline$\#$ & $\boldsymbol{p}_{\text {BLRT }}$ & $\boldsymbol{p}_{\text {VLMR }}$ & AIC & BIC & aBIC & E & $<5 \%$ \\
\hline 2 & $<.001$ & $<.001$ & 12833.264 & 12931.772 & 12871.418 & 0.787 & 0 \\
\hline $3^{*}$ & $<.001$ & .055 & 12403.519 & 12538.319 & 12455.729 & 0.777 & 1 \\
\hline 4 & $<.001$ & .392 & 12228.291 & 12399.384 & 12294.558 & 0.817 & 1 \\
5 & $<.001$ & .201 & 12089.744 & 12297.129 & 12170.067 & 0.784 & 2 \\
\hline
\end{tabular}

Anmerkungen: $\quad$ = Anzahl latenter Profile; $p_{\text {BLRT }}=\mathrm{p}$-Wert des Bootstrap-Likelihood-Ratio-Test; $p_{\mathrm{LMR}}=\mathrm{p}$-Wert des adjustierten Vuong-Lo-Mendell-Rubin-Test; AIC = Akaike Information Criterion; $\mathrm{BIC}=$ Bayesian Information Criterion; $\mathrm{aBIC}=$ stichproben-adjustiertes BIC; E= Entropie; $<5 \%=$ Anteil an Klassen mit weniger als $5 \%$ der Fälle; ${ }^{*}=$ gewählte Lösung.

Profillösung ausgewählt. Die mittleren Klassenzugehörigkeitswahrscheinlichkeiten waren sehr gut (.87-.93).

Dem ersten Profil wurden 63 (4,8\%) der Studierenden zugeordnet. Diese Gruppe war durch ein vergleichsweise niedrigeres Zustimmungsniveau zu allen Rollen (3.69 $\leq M \leq 4.55)$ charakterisiert. Das zweite Profil umfasste 555 $(42,1 \%)$ Studierende, für die ein relativ hohes Niveau der Zustimmung zu allen Lehrkraftrollen charakteristisch war $(4.37 \leq M \leq 5.21)$. Das dritte Profil umfasste 701 (53.1\%) Studierende, für die ein durchweg sehr hohes Niveau der $\mathrm{Zu}$ stimmung zu allen Lehrkraftrollen bezeichnend war $(5.08 \leq M \leq 5.68)$. Die größten Unterschiede waren hinsichtlich den Rollen ER $\left(M_{\text {Profil } 1}=3.84\right.$, $\left.S D_{\text {Profil } 1}=0.44 ; M_{\text {Profil }}=5.56, S D_{\text {Profil }}=0.44\right)$ und $\operatorname{HE}\left(M_{\text {Profil }}=4.04, S D_{\text {Profil }}=0.59 ; M_{\text {Profil }}=5.48\right.$, $\left.S D_{\text {Profil }}=0.59\right)$ festzustellen und der kleinste in Bezug auf die Rolle WV $\left(M_{\text {Profil } 1}=4.55, S D_{\text {Profil }}=\right.$ $\left.0.41, M_{\text {Profil }}=5.25, S D_{\text {Profil }}=0.41\right)$.

\section{Unterschiede zwischen den identifizierten Subgruppen}

Im nächsten Schritt wurde untersucht, ob sich die Studierenden der identifizierten Profile hinsichtlich relevanter Hintergrundmerkmale (Studiengang, Studiendauer, Geschlecht) und hinsichtlich ihrer bisherigen praktischen Erfahrungen voneinander unterscheiden (Fragestellung 3). Die Ergebnisse des Chi-Quadrat-Tests ergaben, dass der Unterschied in der Verteilung auf die Schularten zwischen den drei Profilen statistisch signifikant wurde $\left(\chi^{2}(4, N=1319)=42.87\right.$, $p<.001$, Cramér's $V=.13)$. Profil 1 enthielt mehr Gymnasiallehramtsstudierende $\left(8,8 \%{ }^{5} ; z=3.4\right.$; $p<.05$, Bonferroni-korrigiert), als zu erwarten wäre. In Hinblick auf die Studiendauer (Anzahl der studierten Semester) ließen sich keine Unterschiede zwischen den latenten Profilen nachweisen. Die Geschlechterverteilung unterschied sich signifikant zwischen den Gruppen $\left(\chi^{2}(2, N=1319)=42.15, p<.001\right.$, Cramér's $V=$ .18). Profil $1\left(10,2 \%^{6} ; z=3.7 ; p<.01\right.$, Bonferroni-korrigiert) und Profil $2(54,2 \% ; z=2.8$; $p<.05$, Bonferroni-korrigiert) wiesen einen signifikant höheren Anteil männlicher Studierender auf; Profil 3 hingegen einen signifikant niedrigeren Anteil $(35,6 \% ; z=-3.6 ; p<.01$, Bonferroni-korrigiert), als zu erwarten gewesen wäre.

Die Untersuchung der Unterschiede hinsichtlich der praktischen Erfahrungen basierte designbedingt auf Teilstichproben $\left(N_{\text {Pficht }}=302\right.$, $N_{\text {Zuart }}=428$ ), wobei die Zugehörigkeitsquoten zu den Profilen proportional zur Gesamtstichprobe abgebildet wurden (Profil 1: 4\%, Profil 2: 44\%, Profil 3: 52\%). In Hinblick auf die Anzahl der absolvierten Pflichtpraktika ließen sich keine signifikanten Unterschiede zwischen den Profilen nachweisen. Hinsichtlich der berichteten Anzahl zusätzlicher Praxiserfahrungen zeigte sich ein signifikanter Effekt $(F(2,425)=3.30$, $\left.p<.05, \eta^{2}=.02\right)$. Profil-3-Studierende gaben tendenziell mehr zusätzliche Erfahrungen an als andere Studierende $(t(425)=-2.32, p<.10$, Bonferroni-korrigiert). Auch für die Frage, wie hilfreich diese Erfahrungen für die Reflexion und Anwendung des Theoriewissens war, zeigte sich ein signifikanter Effekt $(F(2,302)=4.81, p<.01$,

Prozentangaben innerhalb von Schulart.

Prozentangaben innerhalb von Geschlecht. 
$\left.\eta^{2}=.03\right)$. Der Mittelwert der Profil-3-Studierenden war dabei signifikant höher als der Mittelwert von Profil-2-Studierenden (mittlere Differenz $=0.34, p<.01$, Bonferroni-korrigiert).

Darüber hinaus wurde ein multiplikativer Index aus der Anzahl der zusätzlichen Praxiserfahrungen und der darauf bezogenen Antworten zur Reflexion und Anwendung des Theoriewissens gebildet und mit der Profilzugehörigkeit in Beziehung gesetzt. Die Ergebnisse zeigten einen signifikanten Effekt $(F(2,415)=6.19, p<.01$, $\left.\eta^{2}=.03\right)$. Profil-3-Studierende wiesen signifikant höhere Werte des Indexes zusätzliche Praxiserfahrung auf als Profil-2-Studierende (mittlere Differenz $=1.0, p<.01$, Bonferroni-korrigiert).

\section{Diskussion}

Ein Ziel des Lehramtsstudiums ist es, die Studierenden darin zu unterstützen, ihre berufsrelevanten Vorstellungen sukzessive an die Standards des Berufs anzunähern (vgl. Messner \& Reusser, 2000). Mehrheitlich wurde darauf hingewiesen, dass angehende Lehrkräfte bereits während ihrer eigenen Schulzeit stabile, auf den Lehrkräfteberuf bezogene Überzeugungen entwickeln (vgl. Reusser \& Pauli, 2014; Rothland \& Terhart, 2007). Umso wichtiger erscheint es, die von den Studierenden mitgebrachten Überzeugungen in der Lehrkräftebildung zu beachten (Fives \& Buehl, 2012).

Dieser Beitrag untersuchte sechs Facetten des professionellen Rollenverständnisses zum Beruf der Lehrkraft bei Studierenden mit unterschiedlicher Studiendauer. Ein personenzentrierter Ansatz wurde gewählt, um verschiedene Subgruppen von Lehramtsstudierenden zu identifizieren, die sich im Verhältnis verschiedener Facetten des professionellen Rollenverständnisses unterscheiden.

Die Ergebnisse zeigen, dass die Studierenden alle sechs betrachteten Rollenfacetten durchschnittlich als wichtig bis sehr wichtig einschätzten; Unterschiede zwischen den Facetten waren eher gering ausgeprägt. Ein Vergleich von Studierenden am Beginn und am Ende des Studiums ergab, dass sich die Einschätzungen für die Rolle Mediator/Mediatorin für selbstständige Lernprozesse (ML) sowie Beurteiler/Beurteilerin (BEU) zwischen Studierenden aus den verschiedenen Studienabschnitten signifikant unterschieden. ML und BEU wurden von Studierenden mit fortgeschrittener Studiendauer als signifikant wichtiger wahrgenommen als von Studienanfängerinnen und -anfängern. Die Befunde sind dabei nur teilweise in Einklang mit den theoretischen Erwartungen, wonach verbreitete Vorstellungen zur Lehrkräfterolle, wie z. B. Wissensvermittlung und Beurteilung, an Wichtigkeit verlieren sollten, und andere Aufgabenfelder, wie z. B. das Koordinieren selbstständiger Lernprozesse und Beraten, eine Aufwertung erfahren sollten (vgl. Messner \& Reusser, 2000).

Insgesamt waren also für zwei von sechs Rollen Unterschiede zwischen den Kohorten mit kleiner Effektstärke nachweisbar, wobei das Gesamtbild geprägt war von Geschlechts- und Schulartunterschieden, die schon zu Beginn der Ausbildung bestanden. Grundschullehramtsstudierende wiesen der Rolle Mediator/Mediatorin für selbstständige Lernprozesse, die einem schülerzentrierten konstruktivistischen Unterrichtsansatz entspricht, sowie Beratungs- und Beurteilungsaufgaben eine höhere Wichtigkeit zu. Dieser Befund steht im Einklang mit bisherigen empirischen Ergebnissen zu schulartspezifischen Anforderungen an Lehrpersonen (Weiß et al., 2013). Einen weiteren möglichen Erklärungsansatz für die Unterschiede zwischen den Schularten stellen schulartspezifische berufsbezogene Interessen und Berufswahlmotive der Studierenden dar (vgl. z. B. Retelsdorf \& Möller, 2012). Insgesamt lassen sich die Ergebnisse dahingehend interpretieren, dass Lehramtsstudierende bereits zu Beginn ihres Studiums über ein Rollenverständnis verfügen, in dem sich die Komplexität des Berufs abbildet, und dass sich die Ausprägungen dieser Überzeugungen nur geringfügig verändern. Die Ergebnisse reihen sich somit in den bisherigen Forschungsstand ein, aus dem nur für wenige Facetten Hinweise auf Veränderung während des Studiums hervorgehen (Löfström \& Poom-Valickis, 2013; Saban et al., 2007). 
Um der Komplexität verschiedener Facetten des professionellen Rollenverständnisses gerecht zu werden, wurde ein personenzentrierter Ansatz gewählt, mit dem die Konstellation der Facetten zueinander berücksichtigt wurde. Es konnten drei Subgruppen bzw. Typen von Studierenden mit unterschiedlichen Profilen identifiziert werden. Die identifizierten Profile unterschieden sich im Niveau voneinander, wobei Profil 1 mit vergleichsweise niedrigeren Zustimmungen zu den Rollen 5\% der Befragten angehörten, Profil 2 mit eher hohen Zustimmungen $42 \%$ und Profil 3 mit durchweg sehr hohen Zustimmungen zu allen Lehrkraftrollen 53\%. Die Profile zeigten wenig Differenzierung zwischen den Facetten, auffällig ist die geringe Bedeutungszuschreibung der Rollen Erzieherin/Erzieher und Helferin/ Helfer in Profil 1 (s. Abb. 1). Während für Studiendauer und Anzahl absolvierter Pflichtpraktika keine signifikanten Unterschiede zwischen den Profilen nachgewiesen werden konnten, zeigten sich für Geschlecht, Schulform und für zusätzliche Praxiserfahrungen und deren Reflexion Effekte. So waren in Profil 1 (eher niedrige Ausprägungen) mehr Gymnasiallehramtsstudierende und mehr männliche Studierende, als zu erwarten gewesen wäre. Hingegen gaben Studierende in Profil 3 (sehr hohe Ausprägungen) tendenziell mehr zusätzliche praktische Erfahrungen an und bewerteten diese als signifikant hilfreicher für die Reflexion als Studierende in Profil 2 (eher hohe Ausprägungen). Die Interpretation dieses Befundes ist nicht eindeutig. Vor dem Hintergrund der geringen Unterschiede zwischen den Studienabschnitten ließe sich vermuten, dass Studierende, die alle Rollenfacetten als sehr wichtig beurteilen, engagierter und reflektierter sind. Ebenso könnten vielfältige reflektierte praktische Erfahrungen die Komplexität des Aufgabenfeldes einer Lehrkraft für die Studierenden erfahrbar machen und so zu sehr hohen Wichtigkeitseinschätzungen in allen Facetten führen. Diese Sichtweise steht im Einklang mit theoretischen Annahmen, wonach Reflexion und bewusste Auseinandersetzung mit den eigenen Überzeugungen und Erfahrungen wichtige Voraussetzungen für eine Professionalisierung sind (vgl.
Philip, 2007; Reusser \& Pauli, 2014). Praktische Erfahrungen können sehr unterschiedliche Wirkungen auf die Professionalisierung von Studierenden haben (Keller-Schneider, 2018). In der Literatur wird in diesem Zusammenhang die zentrale Bedeutung einer curricular systematisch eingebundenen Förderung von theoriegeleiteter Reflexion während Praxisphasen hervorgehoben (z. B. Weyland, 2014).

Auffällig ist, dass Studierende in Profil 2 und 3 durch hohe bis sehr hohe Zustimmungen zu allen Facetten gekennzeichnet sind, was für eine geringe Differenzierung bzw. Profilbildung im Rollenverständnis spricht. Es ist dabei nicht auszuschließen, dass Antworttendenzen der Studierenden die Ergebnisse verzerren und zur Identifikation der Profile auf verschiedenen Niveaus beitragen. Außerdem ist bei der Interpretation der Ergebnisse zu berücksichtigen, dass Selbstselektionsprozesse bei der Aufnahme eines Lehramtsstudiums bzw. der Wahl der Schulart wirksam sind. Die Studierenden unterscheiden sich in Hinblick auf ihre persönlichen Voraussetzungen, z. B. Berufswahlmotivation, Persönlichkeitseigenschaften, Bildungsbiografie. Diese Faktoren wurden in der vorliegenden Studie nicht berücksichtigt, könnten jedoch in zukünftigen Studien einen wichtigen Beitrag zur Erklärung der professionellen Rollenüberzeugungen leisten (vgl. Kunter et al., 2011).

Einschränkend ist anzumerken, dass in der vorliegenden Querschnittsstudie nicht geklärt werden kann, ob und inwieweit das Lehramtsstudium zur Genese des professionellen Rollenverständnisses beiträgt. Die Ergebnisse stehen zudem in Einklang mit einer umgekehrten Wirkrichtung, nach der die Überzeugungen zur Lehrkräfterolle die Nutzung von Lerngelegenheiten beeinflussen. Längsschnittstudien könnten zur Klärung dieser Frage beitragen. Als besonders bedeutsame Einflussfaktoren auf das Rollenverständnis sind Erfahrungen, sozialisationsbedingte Einflüsse und kontextuale Bedingungen zu nennen. Diese Bereiche könnten in zukünftigen Studien umfassender berücksichtigt werden. Besonderes Augenmerk sollte dabei auf die praktischen Erfahrungen im pädagogischen 
Kontext unter Berücksichtigung ihrer Reflexion gelegt werden. Weiterhin ist die Validität der Erfassung des professionellen Rollenverständnisses infrage zu stellen. Die Selbstberichte der Studierenden zeigen für alle Facetten hohe Zustimmungen, die durch soziale Erwünschtheit verzerrt sein könnten. Selbsteinschätzungen bleiben immer subjektiv; künftige Forschung sollte daher an der Weiterentwicklung der Instrumente zur Erfassung des professionellen Rollenverständnisses ansetzen, um die Varianz in den Antworten zu erhöhen. Zudem sind die eigenen Überzeugungen nicht unbedingt bewusst bzw. nicht immer direkt zugänglich (z. B. Reusser $\&$ Pauli, 2014). In zukünftigen Studien könnte der Einsatz indirekter Maße zur Erfassung des Rollenverständnisses zur Lehrkräfterolle einen zusätzlichen Beitrag leisten.

Gänzlich unbeantwortet muss die Frage bleiben, warum bei männlichen und weiblichen Studierenden Unterschiede in den subjektiven Rollenüberzeugungen festgestellt wurden. Dies kann mit den vorliegenden Ergebnissen nicht beantwortet werden. Auch sollten anschließende Untersuchungen prüfen, welche Bedeutung das Rollenverständnis für die Kompetenzentwicklung der Studierenden hat. Zwar weiß man, dass Überzeugungen nicht nur die Nutzung der Lerngelegenheiten des Studiums und der praktischen Phasen, sondern auch die Handlungspraxis im späteren Beruf beeinflussen können (z. B. Dubberke et al., 2008; Fischer \& Ehmke, 2019), für das professionelle Rollenverständnis zum Lehrkräfteberuf wurde dies jedoch noch nicht untersucht. Aus diesem Grund lässt sich auch keine Aussage über besonders adaptive oder ungünstige Rollenausprägungen aus dem bisherigen Forschungsstand ableiten. Dennoch leistet diese Studie einen Beitrag zum Erkenntnisgewinn im Bereich der Untersuchung überfachlicher Merkmale der professionellen Kompetenz von Lehrkräften. Die Entwicklung des professionellen Rollenverständnisses von angehenden Lehrkräften ist noch relativ wenig erforscht, obwohl es sich um ein Konstrukt handelt, das das Verhalten und Erleben in Lern- und Berufskontexten beeinflussen könnte.
Für die universitäre Lehrkräfteausbildung scheint es von besonderer Bedeutung zu sein, die von den Studierenden mitgebrachten Vorstellungen über den Lehrkräfteberuf zu identifizieren und in der Lehre zu berücksichtigen. Aus den Ergebnissen der vorliegenden Studie lässt sich ableiten, dass nicht nur die Reflexion von Erfahrungen im universitären Kontext, sondern auch von solchen aus außeruniversitären Kontexten in der Konzeption der Lehre Beachtung geschenkt werden sollte. Praxiserfahrungen könnten stärker genutzt werden, um die Komplexität des Lehrkräfteberufs in seinen verschiedensten Facetten aufzuzeigen und zu reflektieren. Eine reflexive Praxis, die bereits im Studium ausgebildet wurde, könnte sich auch positiv auf den weiteren Professionalisierungsprozess auswirken.

\section{Literatur}

Alexandrowicz, R. (2008). Wieviel ist „ein bisserl“? Ein neuer Zugang zum BIC im Rahmen von Latent-ClassAnalysen. In J. Reinecke \& C. Tarnai (Hrsg.), Klassifikationsanalysen in Theorie und Praxis (S. 141-165). Münster: Waxmann.

Baumert, J. \& Kunter, M. (2006): Stichwort. Professionelle Kompetenz von Lehrkräften. Zeitschrift für Erziehungswissenschaft, 9, 469-520. http://dx.doi.org/10.1007/ s11618-006-0165-2

Biedermann, H., Brühwiler, C. \& Krattenmacher, S. (2012) Lernangebote in der Lehrerausbildung und Überzeugungen zum Lehren und Lernen. Beziehungsanalysen bei angehenden Lehrpersonen. Zeitschrift für Pädagogik, 58, 460-475.

Biedermann, H., Brühwiler, C. \& Steinmann, S. (2012). Making the impossible possible? Establishing beliefs about teaching and learning during teacher training courses. In J. König (Hrsg.), Teachers' pedagogical beliefs. Definition and operationalisation - connections to knowledge and performance - development and change (S. 37-52). Münster: Waxmann.

Bönsch, M. (1994). Zur Neubestimmung der Lehrerrolle: Zum Verhältnis von Schule und LehrerInnen. Unterrichtswissenschaft, 22, 75-87.

Calderhead, J. (1996). Teachers: Beliefs and Knowledge. In D. C. Berliner (Hrsg.), Handbook of educational psychology (S. 709-725). New York: Macmillan Library Reference USA.

Dubberke, T., Kunter, M., McElvany, N., Brunner, M. \& Baumert, J. (2008). Lerntheoretische Überzeugungen von Mathematiklehrkräften: Einflüsse auf die Unterrichtsgestaltung und den Lernerfolg von Schülerinnen und Schülern. Zeitschrift für Pädagogische Psychologie, 22, 193-206. http://dx.doi.org/10.1024/1010-0652. 22.34.193

Fischer, N. \& Ehmke, T. (2019). Empirische Erfassung eines „messy constructs“: Überzeugungen angehender 
Lehrkräfte zu sprachlich-kultureller Heterogenität in Schule und Unterricht. Zeitschrift für Erziehungswissenschaft, 22, 411-433. http://dx.doi.org/10.1007/s116 18-018-0859-2

Fischer, N., Hammer, S. \& Ehmke, T. (2018). Überzeugungen zu Sprache im Fachunterricht: Erhebungsinstrument und Skalendokumentation. In T. Ehmke, S. Hammer, A. Köker, U. Ohm \& B. Koch-Priewe (Hrsg.) Professionelle Kompetenzen angehender Lehrkräfte im Be reich Deutsch als Zweitsprache (S. 149-184). Münster, New York: Waxmann.

Fives, H. \& Buehl, M. (2012). Spring Cleaning for the "Messy" Construct of Teachers' Beliefs: What Are They Which Have Been Examined? What Can They Tell Us? In S. Graham, K. R. Harris, S. Graham \& T. C. Urdan (Hrsg.), APA educational psychology handbook, Volume 2. Individual differences and cultural and contextual factors (1. Aufl. APA handbooks in psychology Bd. 2, S. 471-500). Washington, DC: American Psychological Association. http://dx.doi.org/10.1037/13274-019

Hagemann, W. \& Rose, F.-J. (1998). Zur Lehrer/innenErfahrung von Lehramts-Studierenden. Zeitschrift für Pädagogik, 44, 7-19

Hesterberg, T.C. (2015). What teachers should know about the bootstrap: Resampling in the undergraduate statistics curriculum. The American Statistician, 69 $371-386$.

Keller-Schneider, M. (2018). Professionalisierung im Praxissemester - Veränderungen von Kompetenzerleben und Überzeugungen. In M. Rothland \& I. Biederbeck (Hrsg.), Praxisphasen in der Lehrerbildung im Fokus der Bildungsforschung. Beiträge zur Lehrerbildung und Bildungsforschung (BzLB, Band 4, S. 67-81). Münster: Waxmann. https://doi.org/10.5281/zenodo.3744793

KMK (2000). Gemeinsame Erklärung des Präsidenten der Kultusministerkonferenz und der Vorsitzenden der Bildungs-und Lehrergewerkschaften sowie ihrer Spitzenorganisationen. Deutscher Gewerkschaftsbund DGB und DBB - Beamtenbund und Tarifunion. Beschluss der Kultusminis terkonferenz vom 5. 10.2000. Zugriff am 15.6.2020 unter https://www.kmk.org/fileadmin/Dateien/ver oeffentlichungen_beschluesse/2000/2000_10_05-Bre mer-Erkl-Lehrerbildung.pdf

KMK (2019). Standards für die Lehrerbildung: Bildungswissenschaften. Beschluss der Kultusministerkonferenz vom 16. 12.2004 i. d. F. vom 16.5.2019. Zugriff am 25.6. 2020 unter https://www.kmk.org/fileadmin/ver oeffentlichungen_beschluesse/2004/2004_12_16Standards-Lehrerbildung-Bildungswissenschaften.pd

Korthagen, F. A. \& Kessels, J. P. (1999). Linking theory and practice: Changing the pedagogy of teacher education. Educational researcher, 28(4), 4-17.

Kunter, M., Baumert, J., Leutner, D., Terhart, E., Seidel, T., Dicke, T., ... Stürmer, K. (2017). Dokumentation der Erhebungsinstrumente der Projektphasen des BilWissForschungsprogramms von 2009 bis 2016. Bildungswissenschaftliches Wissen und der Erwerb professioneller Kompetenz in der Lehramtsausbildung (BilWiss); die Bedeutung des bildungswissenschaftlichen Hochschulwissens für den Berufseinstieg von Lehrkräften (BilWiss-Beruf). GoetheUniversität; Universitätsbibliothek Johann Christian Senckenberg, Frankfurt am Main.

Kunter, M., Baumert, J., Blum, W., Klusmann, U., Krauss, S. \& Neubrand, M. (Hrsg.). (2011). Professionelle Kom petenz von Lehrkräften - Ergebnisse des Forschungsprogramms COACTIV. Münster: Waxmann.
Kunter, M. \& Pohlmann, B. (2015). Lehrer. In E. Wild \& J. Möller (Hrsg.), Pädagogische Psychologie (S. 261281). Berlin, Heidelberg: Springer. http://dx.doi.org/ 10.1007/978-3-642-41291-2 11

Lo, Y., Mendell, N. R. \& Rubin, D. B. (2001). Testing the number of components in a normal mixture. Biome trika, 88, 767-778. http://dx.doi.org/10.1093/bio met/88.3.767

Löfström, E. \& Poom-Valickis, K. (2013). Beliefs about teaching. Persistent or malleable? A longitudinal study of prospective student teachers' beliefs. Teaching and Teacher Education, 35, 104-113. http://dx.doi.org/ 10.1016/j.tate.2013.06.004

Merk, S., Schneider, J., Bohl, T., Kelava, A. \& Syring, M. (2017). Epistemologische Überzeugungen von Lehramtsstudierenden bezüglich pädagogischen Wissens. Gegenstands-, Quellen- und Kontextspezifität. Journal for educational research online, 9, 169-189.

Messner, H. \& Reusser, K. (2000): Die berufliche Entwicklung von Lehrpersonen als lebenslanger Prozess. Beiträge zur Lehrerinnen- und Lehrerbildung, 18, 157-171.

Muthén, L. K. \& Muthén, B. O. (1998-2019). Mplus User's Guide (Eighth Edition). Los Angeles, CA.

Oser, F. \& Blömeke, S. (2012). Überzeugungen von Lehrpersonen. Einführung in den Thementeil. Zeitschrift für Pädagogik, 58, 415-421.

Pajares, M. F. (1992). Teachers' Beliefs and Educational Research: Cleaning Up a Messy Construct. Review of Educational Research, 62, 307-332.

Philipp, R. A. (2007). Mathematics teachers' beliefs and affect. In F. K. Lester (Hrsg.), Second handbook of research on mathematics teaching and learning. A project of the National Council of Teachers of Mathematics (S. 257-315). Charlotte, NC: Information Age Pub.

Reusser, K. (2000). Weiterentwicklung der fachpädagogi schen Rolle von Lehrpersonen. Beiträge zur Lehrerinnen- und Lehrerbildung, 8, 85-86.

Reusser, K. (2006). Konstruktivismus - vom epistemologischen Leitbegriff zur Erneuerung der didaktischen Kultur. In M. Baer, M. Fuchs, P. Füglister, K. Reusser \& H. Wyss (Hrsg.), Didaktik aufpsychologischer Grundlage. Von Hans Aeblis kognitionspsychologischer Didaktik zur modernen Lehr-und Lernforschung (1. Aufl., S. 151-168). Bern: h.e.p. verlag ag.

Reusser, K. \& Pauli, C. (2014). Berufsbezogene Überzeugungen von Lehrerinnen und Lehrern. In E. Terhart, H. Bennewitz \& M. Rothland (Hrsg.), Handbuch der Forschung zum Lehrerberuf (2. Aufl., S. 643-661). Münster, New York: Waxmann.

Retelsdorf, J. \& Möller, J. (2012). Grundschule oder Gymnasium? Zur Motivation ein Lehramt zu studieren. Zeitschrift für Pädagogische Psychologie, 26, 5-17. https://doi.org/10.1024/1010-0652/a000056

Richardson, V. (1996). The Role of Attitudes and Beliefs in Learning to Teach. In J. P. Sikula, T. Buttery \& E Guyton (Hrsg.), Handbook of research on teacher education. A project of the Association of Teacher Educators (2. Aufl., S. 102-119). New York, NY: Macmillan Library Reference USA.

Rost, J. (2004). Lehrbuch Testtheorie - Testkonstruktion. Psychologie Lehrbuch. 2. Aufl. Bern: Huber.

Rothland, M. \& Terhart, E. (2007). Beruf: Lehrer-Arbeitsplatz: Schule. In M. Rothland (Hrsg.), Belastung und Beanspruchung im Lehrerberuf. Modelle, Befunde, Interventionen (1. Aufl., S. 11-31). Wiesbaden: VS Verlag für Sozialwissenschaften | GWV Fachverlage GmbH Wiesbaden. http://dx.doi.org/10.1007/978-3-531-90500-6_2 
Saban, A., Kocbeker, B. N. \& Saban, A. (2007). Prospective teachers' conceptions of teaching and learning revealed through metaphor analysis. Learning and Instruction, 17 123-139. http://dx.doi.org/10.1016/j.learninstruc. 2007.01.003

Staub, F. C. \& Stern, E. (2002). The nature of teachers' pedagogical content beliefs matters for students' achievement gains. Quasi-experimental evidence from elementary mathematics. Journal of Educational Psychology, 94, 344-355. http://dx.doi.org/10.1037//0022-0663.94. 2.344

Terhart, E. (2011). Lehrerberuf und Professionalität. Gewandeltes Begriffsverständnis - neue Herausforderungen. In W. Helsper \& R. Tippelt (Hrsg.), Pädagogische Professionalität (S. 202-224). Weinheim, Basel: Beltz.

Vries, S. de, Jansen, E. P. W. A. \& Grift, W. J. C. M. van de (2013). Profiling teachers' continuing professional development and the relation with their beliefs about learning and teaching. Teaching and Teacher Education, 33, 78-89. http://dx.doi.org/10.1016/j.tate.2013.02. 006

Weiß, S., Kollmannsberger, M. \& Kiel, E. (2013). Sind Förderschullehrkräfte anders? Eine vergleichende Einschätzung von Expertinnen und Experten aus Regelund Förderschulen. Empirische Sonderpädagogik, 5, $167-186$.

Weyland, U. (2014). Schulische Praxisphasen im Studium: Professionalisierende oder deprofessionalisierende Wirkung. Fachhochschule Bielefeld. Zugriff am 12.10.2020 unter http://www.bwpat.de/profil3/weyland_profil3. pdf

Woolfolk Hoy, A., Davis, H. \& Pape, S. J. (2009): Teacher Knowledge and Beliefs. In P.A. Alexander (Hrsg.), Handbook of educational psychology (2. Aufl., S. 715738). New York: Routledge.

\section{Prof. Dr. Jennifer Paetsch}

Otto-Friedrich-Universität Bamberg

Juniorprofessur für Evaluation im Kontext

von Lehrerbildung

Luisenstraße 5

96047 Bamberg

E-Mail: Jennifer.Paetsch@uni-bamberg.de

\section{Daniel Mann}

Donata Mehler

Otto-Friedrich-Universität Bamberg

WegE-Projekt

Luisenstraße 5

96047 Bamberg

E-Mail: Daniel.Mann@uni-bamberg.de

Donata.Mehler@uni-bamberg.de

\section{Prof. Dr. Barbara Drechsel}

Otto-Friedrich-Universität Bamberg

Professur für Psychologie in Schule und Unterricht

Kapuzinerstraße 16

96047 Bamberg

E-Mail: Barbara.Drechsel@uni-bamberg.de 